CORRECTION

\title{
Correction to: Solving patients with rare diseases through programmatic reanalysis of genome-phenome data
}

Leslie Matalonga, Carles Hernández-Ferrer (D), Davide Piscia, Solve-RD SNV-indel working group, Rebecca Schüle, Matthis Synofzik (D, Ana Töpf, Lisenka E. L. M. Vissers (D), Richarda de Voer (D), Solve-RD DITF-GENTURIS, Solve-RD DITF-ITHACA, Solve-RD DITF-euroNMD, Solve-RD DITF-RND, Raul Tonda, Steven Laurie, Marcos Fernandez-Callejo, Daniel Picó, Carles Garcia-Linares, Anastasios Papakonstantinou, Alberto Corvó, Ricky Joshi (D), Hector Diez, Ivo Gut (iD, Alexander Hoischen, Holm Graessner (D), Sergi Beltran (iD), the Solve-RD Consortium

(C) The Author(s), under exclusive licence to European Society of Human Genetics 2021

European Journal of Human Genetics (2021) 29:1466-1469; https://doi.org/10.1038/s41431-021-00934-6

Correction to: European Journal of Human Genetics https://doi.org/ 10.1038/s41431-021-00852-7

In the original publication of the article, consortium author lists were missing in the article. The details are as below.

\section{Solve-RD SNV-indel working group}

Enzo Cohen ${ }^{13}$, Isabel Cuesta ${ }^{14}$, Daniel Danis ${ }^{15}$, Anne-Sophie Denommé-Pichon ${ }^{16,17,18}$, Yannis Duffourd ${ }^{16,18}$, Christian Gilissen ${ }^{5,7}$, Mridul Johari ${ }^{19}$, Steven Laurie ${ }^{1}$, Shuang $\mathrm{Li}^{20}$, Leslie Matalonga', Isabelle Nelson ${ }^{13}$, Sophia Peters ${ }^{21}$, Ida Paramonov ${ }^{1}$, Sivakumar Prasanth $^{22}$, Peter Robinson ${ }^{15}$, Karolis Sablauskas ${ }^{5,7}$, Marco Savarese $^{19}$, Wouter Steyaert ${ }^{5,7}$, Ana Töpf ${ }^{4}$, Joeri K. van der Velde ${ }^{20}$, Antonio Vitobello ${ }^{16}$.

${ }^{13}$ Sorbonne Université, INSERM UMRS_974, Center of Research in Myology, Paris, France.

${ }^{14}$ Institute of Rare Diseases Research, Spanish Undiagnosed Rare Diseases Cases Program (SpainUDP) \& Undiagnosed Diseases Network International (UDNI), Instituto de Salud Carlos III, Madrid, Spain.

${ }^{15}$ Jackson Laboratory for Genomic Medicine, Farmington, CT, USA.

${ }^{16}$ Inserm - University of Burgundy- Franche Comté, UMR1231 GAD Dijon, France.

${ }^{17}$ Dijon University Hospital, Genetics Department, Dijon, France.

${ }^{18}$ Dijon University Hospital, FHU-TRANSLAD, Dijon, France.

${ }^{19}$ Folkhälsan Research Center, University of Helsinki, Helsinki, Finland.

${ }^{20}$ Department of Genetics, Genomics Coordination Center, University Medical Center Groningen, University of Groningen, Groningen, The Netherlands.

\section{Solve-RD DITF-GENTURIS}

Stefan Aretz ${ }^{21,23}$, Gabriel Capella 5,7 , Richarda M. de Voer ${ }^{5,7}$, Gareth Evans $^{24}$, Jose Garcia Pelaez ${ }^{25,26}$, Elke Holinski-Feder ${ }^{27}$, Nicoline Hoogerbrugge $^{5,7}$, Andreas Laner ${ }^{27}$, Carla Oliveira ${ }^{25,26,28}$, Andreas Rump ${ }^{29}$, Evelin Schröck ${ }^{29}$, Anna Katharina Sommer ${ }^{21}$, Verena Steinke-Lange ${ }^{27}$, Iris te Paske ${ }^{5,7}$, Marc Tischkowitz ${ }^{30}$, Laura Valle ${ }^{31}$.

${ }^{21}$ Institute of Human Genetics, University of Bonn, Bonn, Germany.

${ }^{22}$ MRC Centre for Neuromuscular Diseases and National Hospital for Neurology and Neurosurgery, UCL Queen Square Institute of Neurology, London, UK.

${ }^{23}$ Center for Hereditary Tumor Syndromes, University Hospital Bonn, Bonn, Germany.

${ }^{24}$ Division of Evolution and Genomic Sciences, School of Biological Sciences, Faculty of Biology, Medicine and Health, University of Manchester, Manchester, UK

${ }^{25}$ i3S - Instituto de Investigação e Inovação em Saúde, Universidade do Porto, Porto, Portugal.

${ }^{26}$ IPATIMUP - Institute of Molecular Pathology and Immunology of the University of Porto, Porto, Portugal.

${ }^{27}$ University of Munich, Munich, Germany.

${ }^{28}$ Departament of Pathology, Faculty of Medicine, University of Porto, Porto, Portugal.

${ }^{29}$ Universty of Dresden, Dresden, Germany.

${ }^{30}$ Department of Medical Genetics, National Institute for Health Research Cambridge Biomedical Research Centre, University of Cambridge, Cambridge, UK.

${ }^{31}$ Instituto de Investigación de Bellvitge, Hospitalet de Llobregat, Llobregat, Spain. 


\section{Solve-RD DITF-ITHACA}

Siddharth Banka ${ }^{32,33}$, Elisa Benetti ${ }^{34}$, Giorgio Casari ${ }^{35,36}$, Andrea Ciolf $^{37}$, Jill Clayton-Smith ${ }^{32,33}$, Bruno Dallapiccola ${ }^{37}$, Elke de Boer $^{5,6}$, Anne-Sophie Denommé-Pichon ${ }^{16,17,38}$, Kornelia Ellwanger $^{9,39}$ ', Laurence Faivre ${ }^{16,18,40}$, Christian Gilissen ${ }^{5,7}$, Holm Graessner ${ }^{9,39}$, Tobias B. Haack ${ }^{\prime}$, Anna Hammarsjö ${ }^{41}$, Marketa Havlovicova ${ }^{42}$, Alexander Hoischen ${ }^{5,8,33}$, Anne Hugon ${ }^{43}$, Adam Jackson ${ }^{40}$, Tjitske Kleefstra ${ }^{5,6}$, Anna Lindstrand ${ }^{41}$, Estrella LópezMartín ${ }^{14}$, Milan Macek Jr ${ }^{42}$, Leslie Matalonga', Manuela Morleo ${ }^{36}$, Vicenzo Nigro ${ }^{36}$, Ann Nordgren ${ }^{41}$, Maria Pettersson ${ }^{41}$, Michele Pinelli $i^{36}$, Simone Pizzi ${ }^{37}$, Manuel Posada ${ }^{14}$, Francesca Clementina Radio ${ }^{44}$, Alessandra Renieri ${ }^{34,45,46}$, Caroline Rooryck ${ }^{47}$, Lukas Ryba $^{42}$, Martin Schwarz ${ }^{42}$, Marco Tartaglia ${ }^{37}$, Christel Thauvin ${ }^{16,40}$, Annalaura Torella ${ }^{35,36}$, Aurélien Trimouille ${ }^{38}$, Alain Verloes ${ }^{43,48}$, Lisenka Vissers ${ }^{5,6}$, Antonio Vitobello ${ }^{16}$, Pavel Votypka ${ }^{42}$, Klea Vyshka $^{43,48}$, Birte Zurek ${ }^{9,39}$.

${ }^{32}$ Manchester Centre for Genomic Medicine, St Mary's Hospital, Manchester University Hospitals NHS Foundation Trust, Health Innovation Manchester, Manchester, UK.

${ }^{33}$ Manchester Centre for Genomic Medicine, Division of Evolution and Genomic Sciences, School of Biological Sciences, Faculty of Biology, Medicine and Health, University of Manchester, Manchester, UK.

${ }^{34}$ Med Biotech Hub and Competence Center, Department of Medical Biotechnologies, University of Siena, Siena, Italy.

${ }^{35}$ Dipartimento di Medicina di Precisione, Università degli Studi della Campania "Luigi Vanvitelli,", Napoli, Italy.

${ }^{36}$ Telethon Institute of Genetics and Medicine, Pozzuoli, Italy.

${ }^{37}$ Genetics and Rare Diseases Research Division, Ospedale Pediatrico Bambino Gesù, IRCCS, Rome, Italy.

${ }^{38}$ Laboratoire de Génétique Moléculaire, Service de Génétique Médicale, CHU Bordeaux - Hôpital Pellegrin, Place Amélie Raba Léon, Bordeaux Cedex, France.

${ }^{39}$ Centre for Rare Diseases, University of Tübingen, Tübingen, Germany.

${ }^{40}$ Dijon University Hospital, Genetics Department and Centres of Reference for Development disorders and intellectual disabilities, FHU TRANSLAD and GIMI Institute, Dijon, France.

${ }^{41}$ Karolinska Institutet, Solna, Sweden.

${ }^{42}$ Department of Biology and Medical Genetics, Charles University Prague-2nd Faculty of Medicine and University Hospital Motol, Prague, Czech Republic.

${ }^{43}$ Department of Genetics, Assistance Publique-Hôpitaux de Paris Université de Paris, Robert DEBRE University Hospital, 48 bd SERURIER, Paris, France.

${ }^{44}$ Ospedale Pediatrico Bambino Gesù, Rome, Italy.

${ }^{45}$ Medical Genetics, University of Siena, Siena, Italy.

${ }^{46}$ Genetica Medica, Azienda Ospedaliero-Universitaria Senese, Siena, Italy.

${ }^{47}$ Université Bordeaux, MRGM INSERM U1211, CHU de Bordeaux, Service de Génétique Médicale, Bordeaux, France;
${ }^{48}$ INSERM UMR 1141 “NeuroDiderot", Hôpital R DEBRE, Paris, France.

\section{Solve-RD DITF-euroNMD}

Ana Töpf ${ }^{4}$, Jonathan Baets ${ }^{49,50,51}$, Danique Beijer ${ }^{49,50}$, Gisèle Bonne ${ }^{13}$, Enzo Cohen ${ }^{13}$, Judith Cossins ${ }^{52}$, Teresinha Evangelista ${ }^{13}$, Alessandra Ferlini ${ }^{53}$, Peter Hackman ${ }^{54}$, Michael G. Hanna ${ }^{55}$, Rita Horvath $^{56}$, Henry Houlden ${ }^{55}$, Mridul Johari ${ }^{54}$, Jarred Lau ${ }^{57}$, Hanns Lochmüller 1,57,58,59,60, William L. Macken ${ }^{55}$, Francesco 'Musacchia $^{35,36}$, Andres Nascimento ${ }^{61}$, Daniel Natera-de Benito ${ }^{61}$, Vincenzo Nigro ${ }^{35,36}$, Giulio Piluso ${ }^{35}$, Veronica Pini ${ }^{62}$, Robert D. S. Pitceathly ${ }^{55}$, Kiran Polavarapu ${ }^{57,60}$, Pedro M. Rodriguez Cruz ${ }^{52,63}$, Anna Sarkozy ${ }^{62}$, Marco Savarese ${ }^{54}$, Rita Selvatici ${ }^{53}$, Rachel Thompson $^{57}$, Annalaura Torella ${ }^{35,36}$, Bjarne Udd ${ }^{54}$, Liedewei Van de Vondel $^{50,51}$, Jana Vandrovcova ${ }^{55}$, Irina Zaharieva ${ }^{62}$.

${ }^{49}$ Translational Neurosciences, Faculty of Medicine and Health Sciences, UAntwerpen, Antwerp, Belgium.

${ }^{50}$ Laboratory of Neuromuscular Pathology, Institute Born-Bunge, University of Antwerp, Antwerpen, Belgium.

${ }^{51}$ Neuromuscular Reference Centre, Department of Neurology, Antwerp University Hospital, Antwerpen, Belgium.

${ }^{52}$ Neuromuscular Disorders Group, NDCN, Weatherall Institute of Molecular Medicine, John Radcliffe Hospital, Oxford, UK.

${ }^{53}$ Unit of Medical Genetics, Department of Medical Sciences, University of Ferrara, Ferrara, Italy.

${ }^{54}$ Folkhälsan Research Center, University of Helsinki and Tampere Neuromuscular Center, Tampere, Finland.

${ }^{55}$ Department of Neuromuscular Diseases, UCL Queen Square Institute of Neurology and The National Hospital for Neurology and Neurosurgery, London, UK.

${ }^{56}$ University of Cambridge, England, UK.

${ }^{57}$ Children's Hospital of Eastern Ontario Research Institute, Ottawa, Canada.

${ }^{58}$ Division of Neurology, Department of Medicine, The Ottawa Hospital, Ottawa, Canada.

${ }^{59}$ Brain and Mind Research Institute, University of Ottawa, Ottawa, Canada.

${ }^{60}$ Department of Neuropediatrics and Muscle Disorders, Medical Center - University of Freiburg, Faculty of Medicine, Freiburg, Germany.

${ }^{61}$ Neuromuscular Unit, Neuropaediatrics Department, Institut de Recerca Pediàtrica Hospital Sant Joan de Déu, CIBERER, Barcelona, Spain.

${ }^{62}$ Dubowitz Neuromuscular Centre, UCL Great Ormond Street Hospital, London, UK.

${ }^{63}$ Nuffield Department of Clinical Neurosciences, University of Oxford, Oxford, UK.

\section{Solve-RD DITF-RND}

Jonathan Baets ${ }^{50,51,64}$, Peter Balicza ${ }^{65}$, Patrick Chinnery ${ }^{23}$, Alexandra Dürr ${ }^{66,67,68}$, Tobias Haack ${ }^{9}$, Holger Hengel ${ }^{2,3}$, Rita Horvath ${ }^{56}$, 
Henry Houlden ${ }^{55}$, Erik-Jan Kamsteeg ${ }^{5}$, Christoph Kamsteeg ${ }^{5}$, Katja Lohmann $^{69}$, Alfons Macaya ${ }^{70}$, Anna Marcé-Grau ${ }^{70}$, Ales Maver ${ }^{39}$, Judit Molnar ${ }^{65}$, Alexander Münchau ${ }^{69}$, Borut Peterlin ${ }^{71}$, Olaf Riess ${ }^{9,39}$, Ludger Schöls ${ }^{2,3}$, Rebecca Schüle-Freyer ${ }^{2,3}$, Giovanni Stevanin $66,67,68,72,73$, Matthis Synofzik ${ }^{2,3}$, Vincent Timmerman ${ }^{74,75}$, Bart van de Warrenburg ${ }^{6}$, Nienke van $0 s^{6,76}$, Jana Vandrovcova ${ }^{5{ }^{\prime}}$, Melanie Wayand ${ }^{2,3}$, Carlo Wilke ${ }^{2,3}$

${ }^{64}$ Peripheral Neuropathy Research Group, University of Antwerp, Antwerp, Belgium.

${ }^{65}$ Semelweis University Budapest, Budapest, Hungary.

${ }^{66}$ Institut National de la Santé et de la Recherche Medicale (INSERM) U1127, Paris, France.

${ }^{67}$ Centre National de la Recherche Scientifique, Unité Mixte de Recherche (UMR) 7225, Paris, France.

${ }^{68}$ Unité Mixte de Recherche en Santé 1127, Université Pierre et Marie Curie (Paris 06), Sorbonne Universités, Paris, France.

${ }^{69}$ University of Lübeck, Lübeck, Germany.

${ }^{70}$ Hospital Vall d'Hebron, Barcelona, Spain.

${ }^{71}$ University of Ljubljana, Ljubljana, Slovenia.

${ }^{72}$ Institut du Cerveau-ICM, Paris, France.

${ }^{73}$ Ecole Pratique des Hautes Etudes, Paris Sciences et Lettres Research University, Paris, France.

${ }^{74}$ Peripheral Neuropathy Research Group, Department of Biomedical Sciences, University of Antwerp, Antwerp, Belgium.

${ }^{75}$ Institute Born Bunge, Antwerp, Belgium.

\section{The Solve-RD Consortium}

Olaf Riess ${ }^{9,39}$, Tobias B. Haack ${ }^{9}$, Holm Graessner ${ }^{9,39}$, Birte Zurek ${ }^{9,39}$, Kornelia Ellwanger ${ }^{9,39}$, Stephan Ossowski ${ }^{9}$, German Demidov ${ }^{9}$, Marc Sturm $^{9}$, Julia M. Schulze-Hentrich ${ }^{9}$, Rebecca Schüle ${ }^{2,3}$, Christoph Kessler $^{2,3}$, Melanie Wayand ${ }^{2,3}$, Matthis Synofzik ${ }^{2,3}$, Carlo Wilke ${ }^{2,3}$, Andreas Traschütz ${ }^{2,3}$, Ludger Schöls ${ }^{2,3}$, Holger Hengel ${ }^{2,3}$, Peter Heutink $^{2,3}$, Han Brunner ${ }^{5,6,77}$, Hans Scheffer ${ }^{5,77}$, Nicoline Hoogerbrugge $^{5,7}$, Alexander Hoischen $5,7,8$, Peter A. C. 't Hoen ${ }^{7,78}$, Lisenka E. L. M. Vissers ${ }^{5,6}$, Christian Gilissen ${ }^{5,7}$, Wouter Steyaert ${ }^{5,7}$, Karolis Sablauskas $^{5}$, Richarda M. de Voer ${ }^{5,7}$, Erik-Jan Kamsteeg ${ }^{5}$, Bart van de Warrenburg $^{6,76}$, Nienke van Os ${ }^{6,76}$, Iris te Paske ${ }^{5,7}$, Erik Janssen ${ }^{5,7}$, Elke de Boer ${ }^{5,6}$, Marloes Steehouwer ${ }^{5}$, Burcu Yaldiz ${ }^{5}$, Tjitske Kleefstra ${ }^{5,6}$, Anthony J. Brookes ${ }^{79}$, Colin Veal ${ }^{79}$, Spencer Gibson ${ }^{79}$, Marc Wadsley $^{79}$, Mehdi Mehtarizadeh ${ }^{79}$, Umar Riaz ${ }^{79}$, Greg Warren ${ }^{79}$, Farid Yavari Dizjikan ${ }^{79}$, Thomas Shorter ${ }^{\prime 9}{ }^{\prime}$, Ana Töpf ${ }^{4}$, Volker Straub ${ }^{4}$, Chiara Marini Bettolo ${ }^{4}$, Sabine Specht ${ }^{4}$, Jill Clayton-Smith ${ }^{24}$, Siddharth Banka ${ }^{24,32}$, Elizabeth Alexander ${ }^{24}$, Adam Jackson ${ }^{24}$, Laurence Faivre ${ }^{16,17,18,80,81}$, Christel Thauvin ${ }^{16,18,80,81}$, Antonio 'Vitobello ${ }^{16}$, Anne-Sophie Denommé-Pichon ${ }^{16}$, Yannis Duffourd ${ }^{16,18}$, Emilie Tisserant $^{16}$, Ange-Line Bruel ${ }^{16}$, Christine Peyron ${ }^{82,83}$, Aurore Pélissier ${ }^{83}$, Sergi Beltran ${ }^{1,11}$, Ivo Glynne Gut ${ }^{11}$, Steven Laurie ${ }^{11}$, Davide Piscia ${ }^{11}$, Leslie Matalonga $^{11}$, Anastasios Papakonstantinou ${ }^{11}$, Gemma Bullich ${ }^{11}$, Alberto Corvo ${ }^{11}$, Carles Garcia ${ }^{11}$, Marcos Fernandez-Callejo ${ }^{11}$, Carles Hernández ${ }^{11}$, Daniel Picó ${ }^{11}$, Ida Paramonov ${ }^{11}$, Hanns Lochmüller ${ }^{11}$, Gulcin Gumus ${ }^{84}$, Virginie Bros-Facer ${ }^{85}$, Ana Rath ${ }^{86}$, Marc Hanauer ${ }^{86}$, Annie Olry ${ }^{86}$, David Lagorce ${ }^{86}$, Svitlana Havrylenko ${ }^{86}$, Katia Izem ${ }^{86}$ ' Fanny Rigour ${ }^{86}$, Giovanni Stevanin ${ }^{66,67,68,72,73}$, Alexandra Durr ${ }^{67,68,72,87}$, Claire-Sophie 'Davoine ${ }^{67,68,72,73}$, Léna Guillot-Noel ${ }^{67,68,72,73}$, Anná
Heinzmann ${ }^{67,68,72,88}$, Giulia Coarellii,68,72,88, Gisèle Bonne ${ }^{13}$, Teresinha Evangelista $^{13}$, Valérie Allamand ${ }^{13}$, Isabelle Nelson ${ }^{13}$, Rabah Ben Yaou $^{13,89,90}$, Corinne Metay ${ }^{13,91}$, Bruno Eymard ${ }^{13,89}$, Enzo Cohen ${ }^{13}$, Antonio Atalaia ${ }^{13}$, Tanya Stojkovic ${ }^{13,89}$, Milan Macek Jr. ${ }^{42}$, Marek Turnovec ${ }^{42}$, Dana' Thomasová ${ }^{42}$, Radka Pourová Kremliková ${ }^{42}$, Vera Franková ${ }^{42}$, Markéta Havlovicová ${ }^{2}$, Vlastimil Kremlik ${ }^{42}$, Helen Parkinson $^{92}$, Thomas Keane ${ }^{92}$, Dylan Spalding ${ }^{92}$, Alexander Senf ${ }^{92}$, Peter Robinson ${ }^{15}$, Daniel Danis ${ }^{15}$, Glenn Robert ${ }^{93}$, Alessia Costa ${ }^{93}$, Christine Patch $^{93,94}$, Mike Hanna ${ }^{22}$, Henry Houlden ${ }^{95}$, Mary Reilly ${ }^{22}$, Jana Vandrovcova ${ }^{95}$, Francesco Muntoni ${ }^{62,96}$, Irina Zaharieva ${ }^{62}$, Anna Sarkozy ${ }^{62}$, Vincent Timmerman ${ }^{74,75}$, Jonathan Baets ${ }^{50,51,64}$, Liedewei Van de $V^{\prime}$ dondel ${ }^{64,75}$, Danique Beijer ${ }^{64,75}$, Peter de Jonghe ${ }^{51,75}$, Vincenzo Nigro ${ }^{35,36}$, Sandro Banfi ${ }^{35,36}$, Annalaura Torella ${ }^{35}$, Francesco Musacchia ${ }^{35,36}$, Giulio Piluso ${ }^{35}$, Alessandra Ferlini ${ }^{53}$, Rita Selvatici ${ }^{53}$, Rachele Rossi ${ }^{53}$, Marcella Neri ${ }^{53}$, Stefan Aretz ${ }^{21,23}$, Isabel Spier ${ }^{21,23}$ ', Anna Katharina Sommer ${ }^{21}$, Sophia Peters ${ }^{21}$, Carla Oliveira ${ }^{25,26,28}$, Jose Garcia Pelaez ${ }^{25,26}$, Ana Rita Matos ${ }^{25,26}$, Celina São José ${ }^{25,26}$, Marta Ferreira $^{25,26}$, Irene Gullo ${ }^{25,26,28}$, Susana Fernandes ${ }^{25,97}$, Luzia Garrido ${ }^{98}$, Pedro Ferreira ${ }^{25,26,99}$, Fátima Carneiro ${ }^{25,26,28}$, Morris A. Swertz ${ }^{20}$, Lennart Johansson ${ }^{20}$, Joeri K. van der Velde ${ }^{20}$, Gerben van der Vries $^{20}$, Pieter B. Neerincx ${ }^{20}$, Dieuwke Roelofs-Prins ${ }^{20}$, Sebastian Köhler $^{100}$, Alison Metcalfe ${ }^{93,101}$, Alain Verloes ${ }^{43,48}$, Séverine Drunat $^{43,48}$, Caroline Rooryck ${ }^{47}$, Aurelien Trimouille ${ }^{38}$, Raffaele Castello ${ }^{36}$, Manuela Morleo ${ }^{36}$, Michele Pinelli ${ }^{36}$, Alessandra Varavallo ${ }^{36}$, Manuel Posada De la Paz ${ }^{14}$, Eva Bermejo Sánchez ${ }^{14}$, Estrella López Martín ${ }^{14}$, Beatriz Martínez Delgado ${ }^{14}$, F. Javier Alonso García de la Rosa ${ }^{14}$, Andrea Ciolf ${ }^{37}$, Bruno Dallapiccola ${ }^{37}$, Simone Pizzi ${ }^{37}$, Francesca Clementina Radio ${ }^{37}$, Marco Tartaglia ${ }^{37}$, 'Alessandra Renieri ${ }^{34,45,46}$, Elisa Benetti $^{34}$, Peter Balicza ${ }^{102}$, Maria Judit Molnar ${ }^{102}$, Ales Maver ${ }^{103}$, Borut Peterlin ${ }^{103}$, Alexander Münchau ${ }^{104}$, Katja Lohmann ${ }^{104}$, Rebecca Herzog ${ }^{104}$, Martje Pauly ${ }^{104}$, Alfons Macaya ${ }^{105}$, Anna Marcé-Grau ${ }^{105}$, Andres Nascimiento Osorio ${ }^{106}$, Daniel Natera de Benito ${ }^{106}$, Hanns Lochmüller ${ }^{60,107,108}$, Rachel Thompson ${ }^{60,108}$, Kiran Polavarapu ${ }^{60}$, David Beeson $^{63}$, Judith Cossins ${ }^{63}$, Pedro M. Rodriguez Cruz ${ }^{63}$, Peter Hackman ${ }^{109}$ 'Mridul Johari ${ }^{109}$, Marco Savarese ${ }^{109}$, Bjarne Udd $^{109,110,111}$, Rita Horvath ${ }^{112}$, Gabriel Capella ${ }^{113}$, Laura Valle ${ }^{113}$, Elke Holinski-Feder ${ }^{114}$, Andreas Laner ${ }^{114}$, Verena Steinke-Lange ${ }^{114}$, Evelin Schröck ${ }^{115}$, Andreas Rump ${ }^{115,116}$

${ }^{76}$ Department of Neurology, Radboud University Medical Center, Nijmegen, The Netherlands.

${ }^{77}$ Department of Clinical Genetics, Maastricht University Medical Centre, Maastricht, The Netherlands.

${ }^{78}$ Center for Molecular and Biomolecular Informatics, Radboud University Medical Center, Nijmegen, The Netherlands.

${ }^{79}$ Department of Genetics and Genome Biology, University of Leicester, Leicester, UK.

${ }^{80}$ Dijon University Hospital, Centre of Reference for Rare Diseases: Development Disorders and Malformation Syndromes, Dijon, France.

${ }^{81}$ Dijon University Hospital, GIMI institute, Dijon, France.

${ }^{82}$ University of Burgundy-Franche Comté, Dijon Economics Laboratory, Dijon, France.

${ }^{83}$ University of Burgundy-Franche Comté, FHU-TRANSLAD, Dijon, France.

${ }^{84}$ EURORDIS-Rare Diseases Europe, Sant Antoni Maria Claret 167 08025, Barcelona, Spain.

${ }^{85}$ EURORDIS-Rare Diseases Europe, Plateforme Maladies Rares, Paris, France. 
${ }^{86}$ INSERM, US14 - Orphanet, Plateforme Maladies Rares, Paris, France.

${ }^{87}$ Centre de Référence de Neurogénétique, Hôpital de la PitiéSalpêtrière, Assistance Publique-Hôpitaux de Paris (AP-HP), Paris, France.

${ }^{88}$ Hôpital de la Pitié-Salpêtrière, Assistance Publique-Hôpitaux de Paris (AP-HP), Paris, France.

${ }^{89} \mathrm{AP}-\mathrm{HP}$, Centre de Référence de Pathologie Neuromusculaire Nord, Est, lle-de-France, Institut de Myologie, G.H. Pitié-Salpêtrière, Paris, France.

${ }^{90}$ Institut de Myologie, Equipe Bases de données, G.H. PitiéSalpêtrière, Paris, France.

${ }^{91}$ AP-HP, Unité Fonctionnelle de Cardiogénétique et Myogénétique Moléculaire et Cellulaire, G.H. Pitié-Salpêtrière, Paris, France.

${ }^{92}$ European Bioinformatics Institute, European Molecular Biology Laboratory, Wellcome Genome Campus, Hinxton, Cambridge, UK.

${ }^{93}$ Florence Nightingale Faculty of Nursing and Midwifery, King's College, London, UK.

${ }^{94}$ Genetic Counselling, Genomics England, Queen Mary University of London, Dawson Hall, EC1M 6BQ London, UK.

${ }^{95}$ Department of Neuromuscular Diseases, UCL Queen Square Institute of Neurology, London, UK.

${ }^{96} \mathrm{NIHR}$ Great Ormond Street Hospital Biomedical Research Centre, London, UK.

${ }^{97}$ Departament of Genetics, Faculty of Medicine, University of Porto, Porto, Portugal.

${ }^{98} \mathrm{CHUSJ}$, Centro Hospitalar e Universitário de São João, Porto, Portugal.

${ }^{99}$ Faculty of Sciences, University of Porto, Porto, Portugal.

${ }^{100}$ NeuroCure Cluster of Excellence, Charité Universitätsklinikum, Charitéplatz 1, 10117 Berlin, Germany.
${ }^{101}$ College of Health, Well-being and Life-Sciences, Sheffield Hallam University, Sheffield, UK.

${ }^{102}$ Institute of Genomic Medicine and Rare Diseases, Semmelweis University, Budapest, Hungary.

${ }^{103}$ Clinical Institute of Genomic Medicine, University Medical Centre Ljubljana, Ljubljana, Slovenia.

${ }^{104}$ Institute of Neurogenetics, University of Lübeck, Lübeck, Germany.

${ }^{105}$ Neurology Research Group, Vall d'Hebron Research Institute, Universitat Autònoma de Barcelona, Barcelona, Spain.

${ }^{106}$ Neuromuscular Disorders Unit, Department of Pediatric Neurology, Hospital Sant Joan de Déu, Barcelona, Spain.

${ }^{107}$ Centro Nacional de Análisis Genómico (CNAG-CRG), Center for Genomic Regulation, Barcelona Institute of Science and Technology (BIST), Barcelona, Spain.

${ }^{108}$ Children's Hospital of Eastern Ontario Research Institute, University of Ottawa, Ottawa, ON, Canada.

${ }^{109}$ Folkhälsan Research Centre and Medicum, University of Helsinki, Helsinki, Finland.

${ }^{110}$ Tampere Neuromuscular Center, Tampere, Finland.

${ }^{111}$ Vasa Central Hospital, Vaasa, Finland.

${ }^{112}$ Department of Clinical Neurosciences, University of Cambridge, Cambridge, UK.

${ }^{113}$ Bellvitge Biomedical Research Institute (IDIBELL), Barcelona, Spain.

${ }^{114}$ Medical Genetics Center (MGZ), Munich, Germany.

${ }^{115}$ Institute for Clinical Genetics, Faculty of Medicine Carl Gustav Carus, Technical University Dresden, Dresden, Germany.

${ }^{116}$ Center for Personalized Oncology, University Hospital Carl Gustav Carus, Technical University Dresden, Dresden, Germany. 